\title{
Percepção dos Desenvolvedores sobre Comunicação em Desenvolvimento de Software
}

\author{
Jessica Garcia ${ }^{1}$, Joyce Oliveira ${ }^{1}$, Vanice Cunha ${ }^{2}$, Christiane Nobre ${ }^{2}$, Giulla \\ Araújo $^{3}$, Eunice Nunes ${ }^{2}$, Cristiano Araújo \\ ${ }^{1}$ Faculdade de Engenharia - Universidade Federal do Mato Grosso (UFMT) \\ Rua Quarenta e Nove, 2367 - Boa Esperança, Cuiabá - MT - Brasil \\ ${ }^{2}$ Instituto de Computação - Universidade Federal do Mato Grosso (UFMT) \\ Rua Quarenta e Nove, 2367 - Boa Esperança, Cuiabá - MT - Brasil. \\ ${ }^{3}$ Tribunal de Contas do Estado de Mato Grosso (TCEMT) \\ Rua Conselheiro Benjamin, 01 - Centro Político Administrativo, Cuiabá - MT - Brasil \\ Jessicagarcia132012@hotmail.com, \\ joyceoliveira@ufmt.br, \{eunice.ufmt, crismac) @gmail.com, \\ \{vanice, christiane\}@ic.ufmt.br, giulladtce.mt.gov.br
}

\begin{abstract}
Communication has been a widely studied factor, because when performed ineffectively it leads to problems that affect the delivery of products and services to customers. In the context of software development, teams with gaps associated with communication are hampered in terms of time, cost and effort. This article investigates, from the perspective of developers, definitions, strategies, tools, factors and attitudes related to communication that affect software development. The results show that meetings, videoconferencing tools, interpersonal relationships and trust between team members are elements that potentialize the communication.
\end{abstract}

Resumo. A comunicação tem sido um fator amplamente estudado, pois quando realizada de maneira ineficaz leva a problemas que afetam a entrega de produtos e serviços aos clientes. No contexto do desenvolvimento de software, as equipes com lacunas associadas a comunicação são prejudicadas em termos de tempo, custo e esforço. Este artigo investiga, sob a perspectiva de desenvolvedores, definições, estratégias, ferramentas, fatores e atitudes relacionados à comunicação que afetam o desenvolvimento de software. Os resultados mostram que reuniões, ferramentas de videoconferência, relações interpessoais e a confiança entre os membros da equipe são elementos que potencializam a comunicação.

\section{Introdução}

O processo de desenvolvimento de aplicações realizado por equipes é influenciado por um conjunto de fatores técnicos, de negócio e humanos que caracterizam a natureza da engenharia de software [Wende et al, 2013]. Entre os fatores humanos inerentes às equipes de desenvolvimento de software se destaca a comunicação

Al-Ani e Edwards (2008) definiram comunicação como "atividade ou processo de expressar ideias e sentimentos ou de dar informações às pessoas”. Yangüe et al. (2016) e 
Khan et al. (2015) ressaltam que a comunicação é a função principal usada para trocar as informações entre os membros da equipe. Hsu et al. (2012) afirmam que a comunicação consiste no processo de transferência de informações entre os membros da equipe. Santos et al. (2014) argumentam que a comunicação flui da fonte para o receptor, resultando em transferência e compreensão de um significado" e complementa, apoiando-se em Dierkes et al. (2003), que este processo só é efetivo se o destinatário puder absorver o conteúdo da mensagem e utilizá-lo.

Thissen et al. (2007) e Staton (2017), afirmam que quando a comunicação é realizada ineficazmente prejudica o andamento das ações de rotina e cria conflitos entre as equipes de software. De acordo com Misnevs e Demiray (2017), lacunas na comunicação geram problemas que afetam a entrega de produtos e serviços aos clientes. As tarefas podem ser erroneamente alocadas e, consequentemente, mal realizadas prejudicando tempo, custo e esforço relacionados ao desenvolvimento de software [Santos et al, 2014; Schmidt e Meures, 2016; Rutz e Tanner, 2016].

Existem várias interferências que podem limitar a compreensão da mensagem no ato da comunicação, podendo impedi-la por completo, filtrar parte dela ou ainda atribuir um sentido errôneo [Dias, 2013]. Segundo David (1995), o nível de comunicação é frequentemente criticado como insuficiente ou incompleto em diversas estruturas organizacionais. Pessoas, geralmente, acreditam que se comunicam adequadamente. Contudo, o funcionamento como um todo é considerado deficiente.

Em um estudo feito por Dias (2013) considerando fatores humanos em equipes de engenharia de software, para que a comunicação seja efetiva em projetos existe a necessidade, prioritariamente, de comunicações horizontais flexíveis mesmo em uma organização hierárquica. Sendo assim, uma efetiva comunicação entre equipes é essencial para um esforço colaborativo em engenharia de software [Misnevs e Demiray, 2017].

Cramton e Webber (2005) examinaram como a dispersão geográfica afeta a comunicação em equipes de desenvolvimento de software e utilizaram a perspectiva dos sistemas sociotécnicos para orientar a exploração dessa questão no artigo. Khan et al. (2015) apresentam uma estrutura que mostra o efeito das distâncias geográficas, socioculturais e temporais na comunicação durante o processo de Gerenciamento de Mudanças de Requisitos (RCM) no Desenvolvimento Global de Software (GSD). Com esse estudo eles concluíram que esses três fatores têm um forte impacto negativo no processo de comunicação.

Por sua vez, Graziotin et al. (2018) investigaram que a felicidade é um fator que influencia positivamente a comunicação levando a formas econômicas de melhorar as condições e o desempenho no trabalho, além da diminuição da ocorrência de distúrbios psicológicos. Mishra et al. (2012) observaram o efeito de diferentes elementos do ambiente físico na comunicação, coordenação e colaboração. De acordo com este estudo, um ambiente de trabalho aberto com apenas barreiras de vidro a meia altura e espaço comum desempenha um papel importante na comunicação entre os membros da equipe.

Este artigo tem como objetivo geral analisar a relevância da comunicação para o sucesso de projetos de desenvolvimento de software. Como objetivos específicos, buscase identificar: i) a compreensão de desenvolvedores sobre o termo 'comunicação'; ii) as estratégias associadas à comunicação, iii) a investigação dos fatores que afetam a 
comunicação, iv) as ferramentas que auxiliam o processo da comunicação e v) fatores e atitudes dos desenvolvedores ante situações relacionadas a comunicação.

Este artigo busca se diferenciar dos estudos descritos anteriormente, por se concentrar especificamente na comunicação e por apresentar a visão de especialistas sobre os elementos como definições, estratégias, ferramentas, fatores e atitudes relacionados à comunicação no contexto de desenvolvimento de software.

O artigo está estruturado da seguinte forma: a seção 2 apresenta a metodologia usada para investigar a comunicação na perspectiva dos desenvolvedores; a seção 3 apresenta os resultados e discussões, que agregam respostas às questões de pesquisa sob a ótica de desenvolvedores com comparações sobre o que a literatura diz a respeito do tópico em discussão; na seção 5 são apresentadas as conclusões do estudo realizado.

\section{Metodologia}

O presente estudo foi conduzido por meio de uma pesquisa de opinião, que de acordo com Pfleeger e Kitchenham (2001) consiste em um método de pesquisa que possui por principal técnica de coleta de dados questionários ou checklists que compõe uma estratégia abrangente para descrever, comparar ou explicar conhecimentos, atitudes e os comportamentos a partir dos dados obtidos. Esse método foi escolhido por ser amplamente utilizado para coletar informações e possibilitar o estudo de um fenômeno sob a ótica de sujeitos que, rotineiramente, estão imersos no contexto investigado.

Como a pesquisa de opinião busca por indivíduos que possuem características definidas previamente, a amostra pode ser caracterizada como não probabilística por julgamento. A amostra por julgamento tem objetivo exploratório e pode ser utilizada em grupos reduzidos de indivíduos. Não faz sentido calcular erro amostral ou número mínimo de participantes neste tipo de amostra.

Dentre os vários profissionais que atuam na computação, os desenvolvedores são os atores envolvidos diretamente no processo de análise, programação e teste em um projeto de desenvolvimento de software. Desta forma, esta pesquisa tem como foco a seleção de desenvolvedores com o objetivo de conhecer a opinião destes profissionais sobre definições, estratégias, ferramentas, fatores e atitudes relacionados à comunicação no contexto de desenvolvimento de software.

Como processo de busca e seleção destes profissionais, foi realizada uma busca em língua portuguesa, na rede social de negócios 'Linkedin'. Nessa rede é possível selecionar a categoria da profissão, sendo que foram identificados 274 profissionais classificados como desenvolvedores. Após essa etapa, foi encaminhado por correio eletrônico em 01/03/2019 um formulário a cada identificado, com prazo de resposta até 30/04/2019. Acredita-se que a pesquisa através da internet possibilita uma maior abrangência e acesso a desenvolvedores sem limitações das fronteiras geográficas, limitando-se apenas pela língua portuguesa.

Para elaboração do formulário, as perguntas foram retiradas da literatura relacionada à comunicação em equipes de desenvolvimento de software. Foram criadas perguntas objetivas com campos adicionais para os desenvolvedores fornecerem sugestões e opiniões. No total, foram definidas 24 questões compostas por cinco alternativas de resposta. 
O formulário disponibilizado aos desenvolvedores possui seis questões demográficas relacionadas ao perfil que englobam o nome do participante, sua formação acadêmica, nome da empresa em que trabalha, quantos anos trabalha na empresa e quanto tempo trabalha na área de desenvolvimento de software. Além disso, o formulário também abordou perguntas sobre o entendimento do participante a respeito da comunicação e sobre os fatores que atuam sobre a comunicação.

O Google Forms foi utilizado para elaborar o formulário. Os dados foram categorizados e analisados por meio de análise temática [Cruzes e Dyba, 2011]. Palavras centrais associadas as respostas foram classificadas por temas que representam a ideia central das opiniões dadas pelos especialistas. As respostas foram tabuladas e categorizadas utilizando técnicas da estatística descritiva. O questionário completo pode ser acessado em goo.gl/forms/pTHXnKah5aXUnzt02

\section{Resultados e Discussões}

Foram enviados 274 convites, sendo que 34 desenvolvedores aceitaram responder o formulário. Dentre os desenvolvedores participantes, cerca de $88 \%$ tem graduação e $12 \%$ pós-graduação, significando que a grande maioria dos profissionais não participaram de estudos mais avançados. A média ponderada de tempo de exercício da atividade está em cerca de cinco anos, sendo que $24 \%$ tem menos de dois anos de trabalho e $21 \%$ tem mais de nove anos de profissão. Cerca de $56 \%$ dos profissionais tem no máximo cinco anos de atividade, sinalizando que a maioria ainda está no início da curva de aprendizagem profissional.

A definição de comunicação é essencial para o entendimento da relevância deste fator no desenvolvimento de software. Com base no conceito de comunicação estratégias podem ser elaboradas para geri-la e fomentá-la na organização. Perguntou-se aos desenvolvedores qual seria o conceito de comunicação:

$>47,06 \%$ dos desenvolvedores se associaram à percepção de Yagüe et al. (2016) que afirmaram que "a comunicação é o processo de transmissão ou troca de informações através da fala, escrita ou meios tecnológicos";

> 14,7\% dos desenvolvedores concordaram com Dullemond et al. (2011) que destacaram que "a comunicação permite o compartilhamento de informações entre as pessoas do mesmo projeto, gerenciando a troca de conhecimento para resolver tarefas e conflitos";

$>11,77 \%$ dos desenvolvedores seguiram o conceito de Vincent (2008), que defende que "a comunicação é o desenvolvimento da compreensão de uma ideia ou conceitos transmitidos entre as pessoas";

11,77\% dos desenvolvedores apoiaram a definição de Mishra et al. (2012) que ressaltaram que "a comunicação é a transferência e compreensão de significados partindo de uma fonte por meio de fala, escrita ou sinais";

$>8,82 \%$ dos participantes indicaram que “a comunicação é definida como um processo de transferência de informações entre os membros da equipe, podendo ser clara ou não." Esta definição também é dada por Guzzi et al. (2012) que afirmam que "a comunicação produz a capacidade de processar informações";

$>$ um desenvolvedor de forma objetiva afirmou que a comunicação consiste em "transmitir uma mensagem, independente do meio";

um desenvolvedor se absteve de opinar sobre o conceito de comunicação. 


\subsection{Estratégias para comunicação em equipes de desenvolvimento de software}

Estratégias neste artigo podem ser compreendidas como soluções para melhorar a comunicação.

> $38,23 \%$ dos desenvolvedores afirmaram que a estratégia mais eficiente de comunicação em desenvolvimento de software consiste em reuniões com toda equipe. Misnevs e Demiray (2017) também destacam a eficiência desta estratégia por facilitar a percepção de sinais não verbais como linguagem corporal e expressões faciais.

> $23,53 \%$ dos desenvolvedores alegam que uma boa estratégia é a comunicação por ferramentas que minimizem a distância geográfica, como de videoconferência e de mensagens instantânea. Bano et al. (2016) corroboram a importância dessa estratégia principalmente quando a equipe é geograficamente dispersa.

$>20,59 \%$ dos desenvolvedores ressaltaram que troca de informações formais, ou seja, por meio de relatórios, atas, códigos comentados também consiste em uma boa estratégia de comunicação. Esta é uma das estratégias investigadas por Guzzi et al. (2012), que inclusive propõe uma ferramenta que foca na inserção de comentários no código fonte como meio de comunicação.

$17,65 \%$ dos desenvolvedores afirmaram que conversas presenciais e informais entre os membros da equipe também consiste em uma ferramenta estratégica visto que a informalidade dá abertura para troca de experiências e aprendizado. Korkala e Maurer (2014) concordam com essa perspectiva e afirmam que a comunicação informal prover rápido feedback e consequentemente, rápida correção de erros.

\subsection{Ferramentas de apoio à comunicação em equipes de desenvolvimento de software}

É classificada como uma ferramenta todo meio automatizado para auxiliar uma parte do processo e suportar tarefas de processo individuais, como verificar a consistência de um projeto, compilar um programa e comparar os resultados dos testes [Sommerville, 2007]. $\mathrm{Na}$ análise do uso de ferramentas de apoio à comunicação, quanto ao uso do correio eletrônico:

$>75,53 \%$ dos desenvolvedores o consideram regular.

> $17,65 \%$ dos desenvolvedores o acham ruim; o motivo da baixa aceitação consiste na demora de feedback e nos riscos envolvidos no recebimento da mensagem, por exemplo, se a caixa do receptor estiver cheia, o correio eletrônico pode não ser recebido.

$>8,82 \%$ dos desenvolvedores o consideram ótimo.

Quanto ao chat como ferramenta, os desenvolvedores:

$>52,94 \%$ acham regular.

$>26,47 \%$ consideram chat e redes sociais ótimas.

$>20,59 \%$ consideram ruim.

Com relação a videoconferência, os desenvolvedores:

$>55,88 \%$ concordam que é uma ótima ferramenta para realização dos projetos.

$>41,18 \%$ acham regular.

$>2,94 \%$ consideraram essa ferramenta ineficaz para o desenvolvimento de software,

Quanto ao telefone, os desenvolvedores perceberam que:

$>70,58 \%$ acham regular como meio de comunicação em equipes de desenvolvimento de software.

$>14,71 \%$ acham ruim. 
$14,71 \%$ acham uma ferramenta ótima.

\subsection{Fatores que influenciam a comunicação em equipes de desenvolvimento de software}

Neste artigo, consideramos fatores os elementos que atuam como barreiras ou facilitadores que impactam a comunicação da forma que são geridos. Entre os desenvolvedores:

$>38,23 \%$ acreditam que o fator que afeta a comunicação em equipes de desenvolvimento de software é a falta de relações interpessoais; esse fator foi observado por Matturro et al. (2015), onde desenvolvedores normalmente possuem personalidade introspectiva, o que dificulta a interação.

$>23,53 \%$ afirmaram rejeição por parte da equipe.

$>23,53 \%$ acreditam que conflitos entre os membros também são fatores que afetam a comunicação no processo de desenvolvimento das aplicações.

> $11,77 \%$ alegaram que a distância física prejudica a comunicação, o que é um ponto interessante visto que segundo Rutz e Tanner (2016), este é um fator amplamente abordado na literatura como um dos mais negativos à comunicação no contexto de equipes de desenvolvimento de software.

$>2,94 \%$ acham que a diversidade cultural consiste em um impedimento à comunicação em equipes de desenvolvimento de software. Esse é um fator abordado por Guzzi et al. (2012) e Wende et al. (2013) que também afirmam que diferentes linguagens, tradições, religiões e políticas afetam a comunicação em equipes de desenvolvimento de software. Os desenvolvedores ainda mencionaram individualismo, ou seja, membros da equipe que não compartilham as informações como um fator negativo à comunicação. De acordo com Iden e Bygstad (2018), este fator ocorre por falta de confiança entre os membros da equipe que podem se sentir ameaçados pela competitividade ou se sentirem inferiorizados ante aqueles que possuem maior conhecimento. $\mathrm{O}$ excesso de informação que acaba diluindo as informações importantes também foi apresentado como fator negativo à comunicação nesse contexto. Schröter et al. (2012) afirmam que neste caso é essencial que a equipe dê enfoque a qualidade da informação de modo que o que é relevante seja priorizado.

A comunicação é tida como um dos aspectos associados aos humanos. A atitude do receptor diante do recebimento da mensagem do emissor em muitos casos é influenciada por motivações psicológicas e comportamentais. Ao serem questionados sobre qual atitude os desenvolvedores tomam ao receber uma informação que não compreendem:

> 50\% afirmaram que inicialmente tentam compreender o conteúdo sozinhos realizando pesquisas; apenas após essa atitude o receptor questiona o emissor da mensagem.

$>41,18 \%$ dos desenvolvedores afirmaram que imediatamente questionam o emissor da mensagem.

$>5,88 \%$ questionam outra pessoa da equipe com quem possuem maior afinidade.

$2,94 \%$ tentam compreender sozinhos e não questionam o emissor da mensagem; é perceptível que a falta de confiança e o receio de ser inferiorizado impedem que os desenvolvedores questionem os receptores, o que pode levar à interpretação errônea da mensagem e, consequentemente, a falhas e retrabalhos no processo de desenvolvimento [Misnevs e Demiray, 2017].

\subsection{Implicações teóricas e práticas}

A comunicação é um fator mediador fortemente vinculado à coordenação e controle da equipe e é essencial para qualquer esforço colaborativo bem-sucedido [Al-Ani e Edwards, 2008; Thissen et al., 2007; Fernando et al., 2011; Korkala et al., 2010]. Dadas essas características, a comunicação eficaz é um dos principais contribuintes para o 
sucesso dos projetos de desenvolvimento de software [Kluender et al., 2017, Prenner et al., 2018].

Nesse contexto, a incapacidade dos membros da equipe de coordenar e comunicar de maneira suficiente e eficaz leva a três grandes problemas com a produção de software: atrasos na entrega, requisitos mal definidos e esforços repetidos de desenvolvimento [Nordio et al., 2011; Marczak e Damian, 2011; Taweel et al., 2009]. A comunicação não elaborada e de baixa qualidade (ou sua perda) são os principais motivos para a falha do projeto de software. Esses pontos levam a uma falta de alinhamento às informações organizacionais e técnicas, à baixa qualidade do processo adotado para a produção, à redução do andamento do projeto e ao aumento do custo do software [de Farias Junior et al., 2016; Khan et al., 2015; Damian et al., 2013; Niinimaki et al., 2009].

Considerando os argumentos acima mencionados, este artigo tem como principal implicação prática a identificação de definição, estratégias, ferramentas, fatores e atitudes relacionados à comunicação sob a visão de desenvolvedores que rotineiramente estão imersos em equipes que realizam atividades de desenvolvimento de software.

Esse resultado pode ser útil a nível prático para desenvolvedores e gestores interessados em entender os problemas na equipe de desenvolvimento de software, gerados pela comunicação ineficiente, tomando-os como ponto de partida para criar estratégias de mitigação e transformá-los em facilitadores. A nível teórico, os resultados contribuem para o aumento do conhecimento da área.

\section{Conclusões}

Este artigo investigou definições, estratégias, ferramentas, fatores e atitudes associadas a comunicação em equipes de desenvolvimento de software sob a visão de desenvolvedores. Foi formulado um conceito no qual a comunicação é caracterizada como um processo de transmissão de ideias, pensamentos, informações, opiniões e sentimentos, independentemente do método utilizado - falado, escrito ou por sinais -, que visa alcançar um entendimento entre os pares. Concluiu-se também que, de acordo com os desenvolvedores que participaram da pesquisa, a estratégia de comunicação mais eficiente é a realização de reuniões com toda equipe.

Com relação as ferramentas, os desenvolvedores consideraram que recursos de videoconferência são os mais precisos no apoio à comunicação em equipes de desenvolvimento de software. Os desenvolvedores acreditam que o fator de maior negatividade à comunicação é a falta de relações interpessoais, o que contradiz a literatura, que amplamente investiga a dispersão geográfica como fator que mais afeta a comunicação no contexto mencionado. Os participantes afirmaram que ao não compreenderem uma informação, a maioria tenta interpretá-la sem ajuda.

Uma porcentagem ainda afirmou que mesmo sem entender a mensagem, não pede ajuda ao receptor ou às pessoas próximas para compreendê-la, o que pode levar a erros e a retrabalho no processo de construção de software. Percebe-se, portanto, que há vários fatores humanos, seja com relação a atitudes do destinatário da mensagem, seja referente à reação do receptor, que impactam a comunicação e, consequentemente, a eficácia do desenvolvimento de software. Características comportamentais e psicológicas são ricas fontes a serem exploradas para uma maior compreensão acerca da influência destes aspectos sobre o indivíduo e sua atuação enquanto membro de uma equipe. Os autores 
agradecem a parceria firmada entre a UFMT, a Uniselva e o TCE-MT que possibilitou a realização da pesquisa

\section{Referências bibliográficas}

Al-Ani, B. and Edwards, H.K. 2008. "A comparative empirical study of communication in distributed and collocated development teams", in 2008 IEEE International Conference on Global Software Engineering, IEEE, August, pp.35-44.

Bano, M., Zowghi, D., \& Sarkissian, N. 2016. "Empirical study of communication structures and barriers in geographically distributed teams". IET Software, 10(5), 147153

Cramton, C. D., \& Webber, S. S. 2005. "Relationships among geographic dispersion, team processes, and effectiveness in software development work teams". Journal of Business Research, 58(6), 758-765

Cruzes, D. S., \& Dyba, T. 2011. "Recommended steps for thematic synthesis in software engineering". In 2011 international symposium on empirical software engineering and measurement, IEEE. September, pp. 275-284.

Damian, D., Helms, R., Kwan, I., Marczak, S. and Koelewijn, B. 2013. "The role of domain knowledge and cross-functional communication in socio-technical coordination", in 2013 35th International Conference on Software Engineering (ICSE), IEEE, May, pp.442-451.

David, W. 1995. Managing Company-Wide Communication, Chapman \& Hall, London, United Kingdom. de Farias Junior, I., Marczak, S., Santos, R., \& Moura, H. 2016. Communication in Distributed Software Development: A Preliminary Maturity Model. In 2016 IEEE 11th International Conference on Global Software Engineering (ICGSE). IEEE. August. pp. 164-173.

Dias, F.N.M. 2013. "O impacto do crescimento de equipes de software nos fatores individuais e nos processos em grupo: Um estudo de caso", Dissertação de mestrado. Universidade Federal de Pernambuco.

Dullemond, K., van Gameren, B., \& van Solingen, R. 2011. An exploratory study on open conversation spaces in Software Engineering. In 7th International Conference on Collaborative Computing: Networking, Applications and Worksharing (CollaborateCom)IEEE. October. pp. 307-316.

Fernando, B.A.J., Hall, T. and Fitzpatrick, A. 2011. "The impact of media selection on stakeholder communication in agile global software development: a preliminary industrial case study", in Proceedings of the 49th SIGMIS Annual Conference on Computer Personnel Research, ACM, May, pp.131-139

Graziotin, D., Fagerholm, F., Wang, X., \& Abrahamsson, P. 2018. "What happens when software developers are (un) happy". Journal of Systems and Software, 140, 32-47.

Guzzi, A., Begel, A., Miller, J. K., \& Nareddy, K. 2012. "Facilitating enterprise software developer communication with CARES”. In 2012 28th IEEE International Conference on Software Maintenance (ICSM). September. pp. 527-536. IEEE. 
Hsu, J.S.C., Shih, S.P., Chiang, J.C. and Liu, J.Y.C. 2012. 'The impact of transactive memory systems on IS development teams' coordination, communication, and performance', International Journal of Project Management, v.30, n.3, pp.329-340.

Iden, J. and Bygstad, B. 2018. "The social interaction of developers and IT operations staff in software development projects", International Journal of Project Management, Vol. 36, No. 3, pp.485-497.

Korkala, M., Pikkarainen, M., \& Conboy, K. 2010. (June). “A case study of customer communication in globally distributed software product development". In Proceedings of the 11th International Conference on Product Focused Software (pp. 43-46). ACM.

Korkala, M. and Maurer, F. 2014. "Waste identification as the means for improving communication in globally distributed agile software development", Journal of Systems and Software, 1th edition Vol.95, pp.122-140.

Khan, A. A., Keung, J., Hussain, S., \& Bennin, K. E. 2015. (April). Effects of geographical, socio-cultural and temporal distances on communication in global software development during requirements change management a pilot study. In 2015 International Conference on Evaluation of Novel Approaches to Software Engineering (ENASE) (pp. 159-168). IEEE.

Kluender, J., Unger-Windeler, C., Kortum, F. and Schneider, K. 2017. "Team meetings and their relevance for the software development process over time", in 2017 43rd Euromicro Conference on Software Engineering and Advanced Applications (SEAA), IEEE, August, pp.313-320.

Marczak, S. and Damian, D. 2011. "How interaction between roles shapes the communication structure in requirements-driven collaboration", in 2011 IEEE 19th International Requirements Engineering Conference, IEEE, August, pp.47-56.

Matturro, G., Raschetti, F., \& Fontán, C. 2015. (May). Soft skills in software development teams: A survey of the points of view of team leaders and team members. In Proceedings of the Eighth International Workshop on Cooperative and Human Aspects of Software Engineering (pp. 101-104). IEEE Press.

Mishra, D., Mishra, A., \& Ostrovska, S. 2012. Impact of physical ambiance on communication, collaboration and coordination in agile software development: An empirical evaluation. Information and software Technology, 54(10), 1067-1078.

Misnevs, B. and Demiray, U. 2017. "The role of communication and metacommunication in software engineering with relation to human errors", Procedia Engineering, Vol. 178, pp.213-222.

Nordio, M., Estler, H. C., Meyer, B., Tschannen, J., Ghezzi, C., \& Di Nitto, E. 2011. (August). How do distribution and time zones affect software development? a case study on communication. In 2011 IEEE Sixth International Conference on Global Software Engineering (pp. 176-184). IEEE.

Niinimaki, T., Piri, A. and Lassenius, C. 2009. "Factors affecting audio and text-based communication media choice in global software development projects", in 2009 Fourth IEEE International Conference on Global Software Engineering, IEEE, July, pp.153-162.4 
Pfleeger, S. L., \& Kitchenham, B. A. 2001. Principles of survey research: part 1: turning lemons into lemonade. ACM SIGSOFT Software Engineering Notes, 26(6), 16-18.

Prenner, N., Klünder, J. and Schneider, K. 2018. 'Making meeting success measurable by participants' feedback', in Proceedings of the 3rd International Workshop on Emotion Awareness in Software Engineering, ACM, June, pp.25-31.

Rutz, L. and Tanner, M. 2016. 'Factors that influence performance in global virtual teams in outsourced software development projects', in 2016 IEEE International Conference on Emerging Technologies and Innovative Business Practices for the Transformation of Societies (EmergiTech), IEEE, August, pp.329-335.

Santos, V., Goldman, A., Martins, D., \& Cortés, M. 2014. (January). The Influence of Organizational Factors on Inter-team Knowledge Sharing Effectiveness in Agile Environments. In 2014 47th Hawaii International Conference on System Sciences (pp. 4729-4738). IEEE.

Schmidt, N., \& Meures, C. 2016. (January). " Mind the Gap": An Analysis of Communication in Agile Global Outsourced Software Development Projects. In 2016 49th Hawaii International Conference on System Sciences (HICSS) (pp. 501-510). IEEE.

Schröter, A., Aranda, J., Damian, D. and Kwan, I. 2012. 'To talk or not to talk: factors that influence communication around changesets', in Proceedings of the ACM 2012 Conference on Computer Supported Cooperative Work, ACM, February, pp.13171326.

Sommerville, I. 2007. Software Engineering, 8ed, v.8, n.9, p.10, Pearson, Add. Wesley.

Taweel, A., Delaney, B., Arvanitis, T.N. and Zhao, L. 2009. 'Communication, knowledge and co-ordination management in globally distributed software development: informed by a scientific software engineering case study', in 2009 Fourth IEEE International Conference on Global Software Engineering, IEEE, July, pp.370-375.

Thissen, M. R., Page, J. M., Bharathi, M. C., \& Austin, T. L. 2007. (April). Communication tools for distributed software development teams. In Proceedings of the 2007 ACM SIGMIS CPR conference on Computer personnel research: The global information technology workforce (pp. 28-35). ACM.

Vincent, M. 2008. (May). Communicating requirements for business: UML or problem frames? In Proceedings of the 3rd international workshop on Applications and advances of problem frames (pp. 16-22). ACM.

Wende, E., Schwabe, G., Philip, T. and King, G. 2013. 'Why they do not understand - a model of knowledge discourse in the transition phase of globally distributed projects', in 2013 46th Hawaii International Conference on System Sciences, IEEE, January, pp.396-405.

Yagüe, A., Garbajosa, J., Díaz, J., \& González, E. 2016. An exploratory study in communication in Agile Global Software Development. Computer Standards \& Interfaces, 48, 184-197. 